\title{
A Study on Meteorological Architecture
}

\author{
Yuqian Wang ${ }^{1,2}$, Yi Yang ${ }^{1,2 *}$ \\ ${ }^{1}$ College of Civil Engineering and Architecture of Zhejiang University \\ ${ }^{2}$ The Architectural Design\&Research Institute of Zhejiang University
}

\begin{abstract}
Under the background of global warming and energy crisis, exploring the new relationship between architecture and climate has always been a hot spot in the construction industry. Thus, a new design pattern "meteorological architecture" guided by a localized meteorological condition is developed .The paper starts with the distinction of the concepts of "meteorology" and "climate" to explains the connotation of "meteorological architecture". Through the analysis of current theories and practices related to meteorological architecture, a new principle of architectural space division and architectural form generation logic have been summarized. Finally, the enlightening effect of meteorological architecture on other fields is proposed.
\end{abstract}

\section{Introduction}

Throughout the history of architecture, there is a long and complicated relationship between architecture and the natural environment. Introducing the concept of meteorology into architecture allows us to understand contemporary architecture from a new perspective.

On the other hand,responding to climate change, saving energy and reducing carbon dioxide emissions has become a global consensus. The high energy consumption of the construction industry has become an important culprit of greenhouse gas emissions. Since the Industrial Revolution, the invention and widespread use of air conditioners and other mechanical equipment has broken the basic balance between people's original construction methods and the natural environment ${ }^{(1)}$. Humans can freely adjust the indoor environment of buildings through these devices. Climate considerations are no longer a necessary part of architectural design. Architecture and nature are in an isolated and opposed relationship. Although air-conditioning equipment does provide people with a "pleasant" living environment to a certain extent, it brings an energy crisis at the expense of consuming a lot of energy.

In the 1940s and 1950s, the rise of the global ecological movement caused us to re-examine the relationship between architecture and climate.Architects kept trying to break the state of isolation between architecture and environment. Concepts such as green buildings, low-carbon buildings, sustainable buildings, passive buildings, and near-zero energy buildings have been put forward one after another.However Some scholars believe that today's energy-saving buildings represented by "energy rating certification" are still a continuation and strengthening of the tradition of isolation from the environment ${ }^{(2)}$. They tried to establish a new relationship between architecture and nature. Thus, a new design pattern "meteorological architecture" guided by a localized meteorological condition is developed.

\section{The Connotation of "Meteorological Architecture"}

What is "Meteorological Architecture"? What is the difference with "climate architecture"? In the past, scholars did not make a strict distinction between "climate" and "meteorology". In fact, there are subtle connections and differences between meteorology and climate. Climate is a natural environmental factor formed during the long-term energy exchange and mass exchange between the earth and the atmosphere. It can also be said to be the long-term average state of meteorological elements. For example, temperate maritime climate, tropical monsoon climate. In the "confrontation" between architecture and climate, people are often subjective responses to the natural environment based on their accumulated life experience. Therefore, in different climate zones, buildings have the same expression, such as thermal insulation culture in cold climates and tropical sun-shading architectural aesthetics. These are all terroir aesthetics formed based on the experience of natural conditions such as climate and geography $^{(3)}$.

Now scholars are more willing to use "meteorological architecture" to explain their architectural practice. Meteorology refers to the general term for various physical phenomena and physical processes in the atmosphere, such as cold, heat, dryness ,humidity, wind, clouds, rain, snow, frost, fog, and lightning. Compared with the study of climate,

\footnotetext{
*Yi, Yang: yyilion@163.com
} 
meteorology pays more attention to the study of the logic of energy operation in nature. Incorporating it into the architectural design, the logic of building space division and building form generation comes from the logic of energy operation in nature, which are also two important directions of current meteorological architecture exploration. The current climate-related global warming and resource consumption are not an issue worthy of continuous concern. It will become the driving force for more innovative initiatives. Compared with the previous use of it as a mandatory additional technical restriction, meteorological architecture is to take the physical elements of pressure, temperature, and relative humidity as a real part of architecture and turn it into a new architectural design tool. Thinking about meteorological architecture means mapping architecture to a brand-new field, establishing a perceptible relationship with space, and treating indoors as an atmospheric environment with abundant climatic conditions, climate changes and climate gradients ${ }^{(4)}$.It is no longer the original climateoriented empirical construction. The meteorological building performs more precise control of the built environment. The research object is reduced from the original broad climatic zone to the small area where the building is located, allowing the building in each different small area more personalized. Among the architect firms currently engaged in meteorological architectural practice, the most representative ones are Philippe Rahm and ROEWU (ROEWU is an designfocused office as a collaboration between Stephen Roe and Chiafang $\mathrm{Wu}$.) Their exploration of the space and form of meteorological architecture has produced a wide range of international influences.

\section{Probe into the Practice of Meteorological Architecture}

\subsection{Exploring the potential of architectural space division}

According to the findings of Rahm's related theories and practices, he is more inclined to explore the potential of using meteorology to divide architectural space. Rahm believes that architecture is not a pile of bricks and cement, but a partitioned reinforced concrete. The purpose of the building is to provide a comfortable living environment. However, most of the problems of modern greenhouse effect and environmental pollution are generated during the construction and use of buildings (5). Rahm's design introduces modern technology to make the atmospheric environment around us participate in the design through physical methods such as sensing, convection, heat conduction, pressure, and radiation. On the basis of following the weather conditions, firstly design a suitable atmospheric environment, then put the building shape in the weather frame, and finally give it different functions according to the needs. This has changed the previous method of mechanically dividing space by function, forming the principle of space organization of " meteorology first, function last".
The House of Convection is one of Rahm's masterpieces of meteorological architecture. The physical term "convection" is often used to describe the combined effect of conduction and fluid flow. In free convection, an increase in temperature causes a decrease in air density, so hot air rises and cold air decreases. The design of the House of Convection is based on the above Archimedes' natural law of warm air rising and cold air falling. In actual situations, different spaces in the room have a certain temperature difference, and the maximum temperature difference between the floor and the ceiling can even reach $10^{\circ} \mathrm{C}$. In addition, the temperature required for different spaces is also different according to different human activities and clothing levels. For example, when we have a quilt on the bed to keep warm, the temperature in the bedroom only needs to be $16^{\circ} \mathrm{C}$; when we sit quietly on the sofa and the human activity is low, the living room usually needs $20^{\circ} \mathrm{C}$; in the kitchen where cooking, the temperature only needs to be maintained At $18^{\circ} \mathrm{C}$; and the bathroom without clothes should be the warmest space in the building. Based on the above indoor weather and human activity data research, arranging functional spaces with different temperature requirements according to the actual temperature difference in the house can effectively save energy. For example, changing the height difference of the horizontal floor, different depths and heights produce different indoor temperatures, and placing the bathroom that needs the most heat at a higher place, and placing the kitchen that does not need heat at a lower place. In this way, the house becomes an energy-saving landscape with different temperatures and scattered patterns. The single and homogeneous indoor space state of the airconditioned building is broken. The logic of energy operation becomes the principle of space division. It can be seen that meteorological architecture requires architects to have an accurate grasp of local meteorological conditions and human physiological characteristics. Meteorological architecture presents a state of interdisciplinary integration.

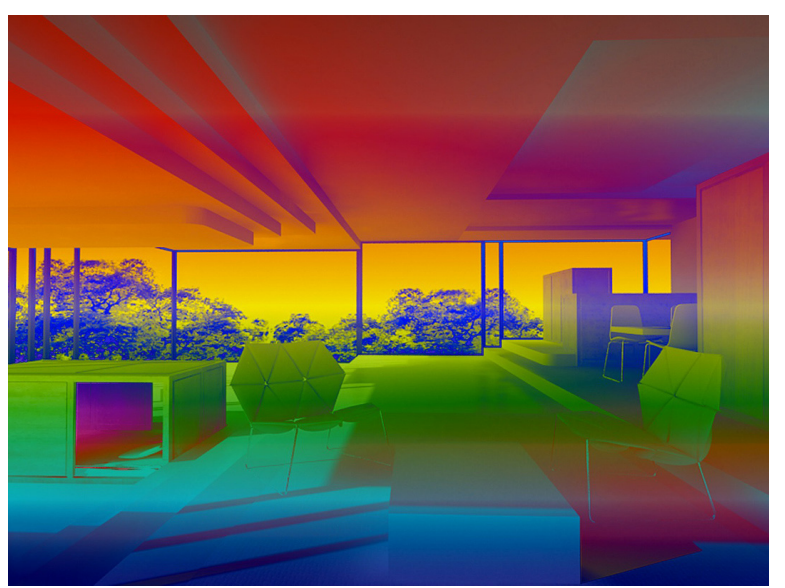

Fig.1.Schematic diagram of cold and warm air exchange 


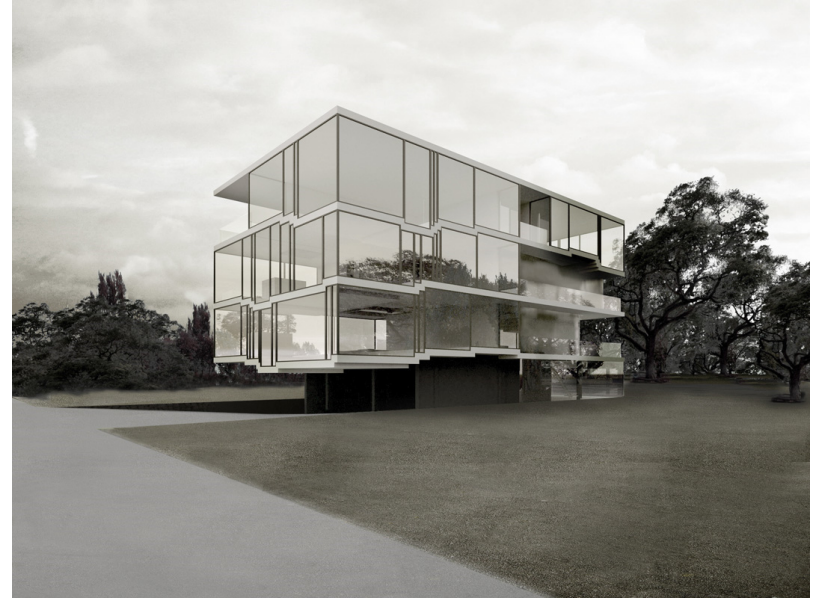

Fig.2. The effect picture of The House of Convection

\subsection{Exploring the innovation of architectural form}

According to the theoretical and practical research of the ROEWU office, although they also involve the exploration of meteorological space, and even coincide with Rahm, their exploration in architectural form innovation is more in-depth. They believe that due to topographical conditions, vegetation conditions, and surrounding buildings each area has a special microclimate. Buildings have the function of regulating wind pressure, wind speed, and solar radiation. These energies are visualized through computer technology, and the logic of energy operation becomes the logic of building form generation.

The book Weather Formations -An Architectural Meteorology which they wrote introduced their theory and practices of meteorological architecture to readers. The author grew up on the northwest coast of Ireland.The stone walls on the coast can stand upright in strong winds for many years. It can be seen that the stones are full of pores, and small air volume can pass through these pores. Therefore, he was inspired to carry out a new structural system for this area. The author uses fluid mechanics simulation to study the relationship between the ratio of holes, wind speed and pressure to explore the stability of the open space, while allowing people to perceive the dynamics of "wind blowing". The author explored a design prototype and their topological relationship. The basic shape are a hexagon and a rectangle. Three vertices are selected as "anchor points". The rule is that the rectangle should be connected with the "anchor points" (the trapezoid is protruding). Then it starts to change. The changed parameters include the angle of rotation and the width of the rectangle. Several dynamic units are generated ${ }^{(6)}$. The wall combines the weight of the material with the lightness of the wind, giving people a sense of sheltering while blowing. Faced with different wind environments, different effects will be obtained by adjusting the parameters and combining different units. The state of isolation between the building and the environment was broken, showing an open state. This new type of structure can be called meteorological architectural structure, showing the power of an architectural "prototype".

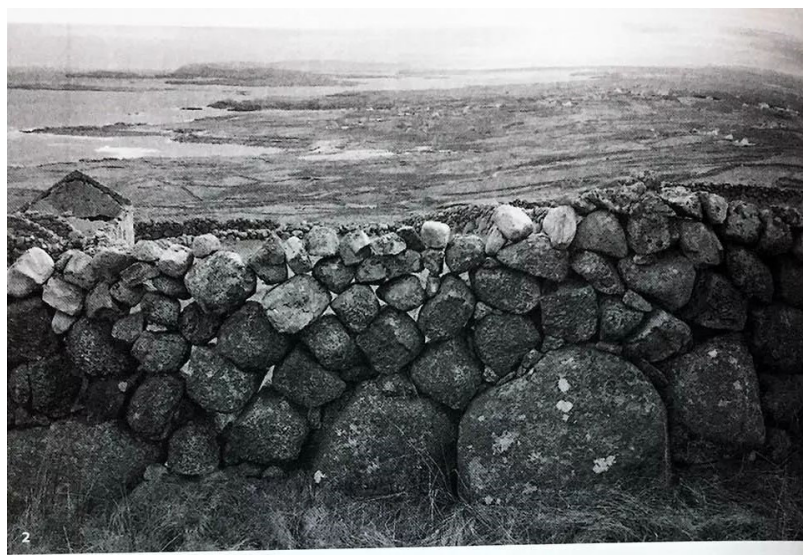

Fig.3.The stone walls on the northwest coast of Ireland

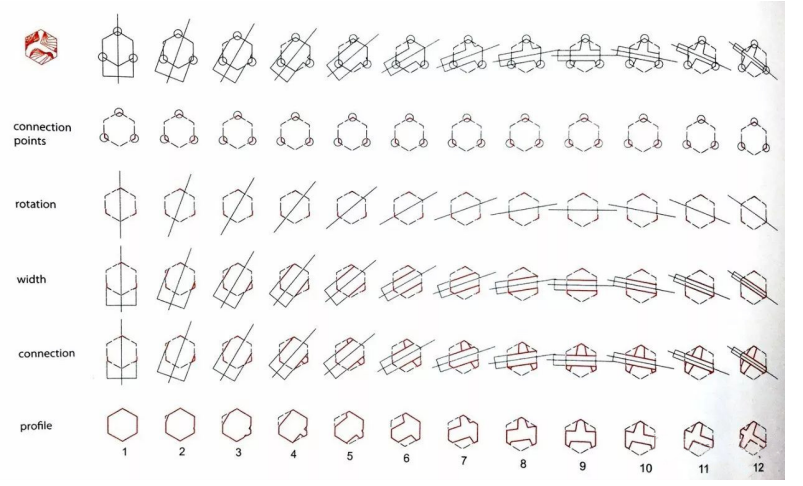

Fig.4. The change process of basic shape

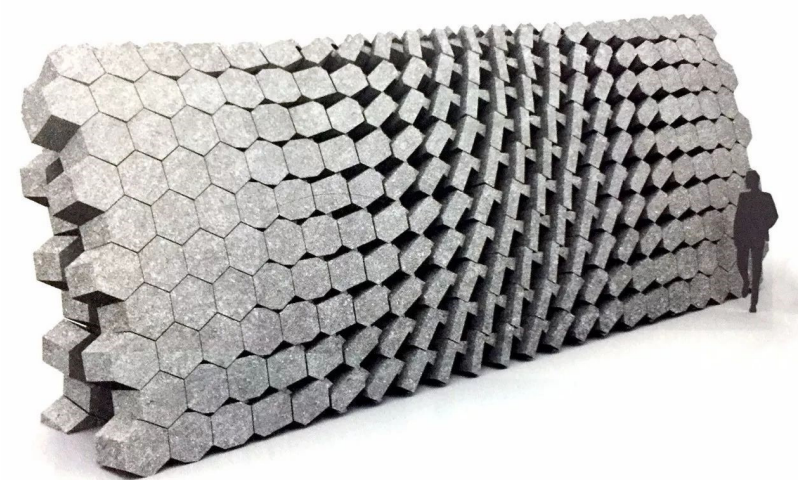

Fig.5. The effect picture of Meteorological material

\section{Enlightenment from Meteorological Architecture}

Although meteorological architecture was developed in the context of the energy crisis, it also has enlightening effects in other fields. In the past 30 years, "healthy buildings" have been a hot spot in the world. In particular, since the new crown virus epidemic, people have been exposed to buildings and their indoor environment for a long time.There are higher requirements for the improvement of building indoor health performance. How to comprehensively promote the physical and mental health of users through the air, water, comfort, fitness, humanities, services and other 
aspects in the building is an important proposition for achieving healthy buildings ${ }^{(7)}$. According to the relevant theories of meteorological architecture, we should start with "building a comfortable climate" as a part of the design. "building a comfortable climate" is a method not a target .Compared with a series of measures to improve the indoor environment after the building is completed, the construction according to this method will greatly improve the health performance of the indoor environment of the building. Meteorological architecture provides new ideas for the development of healthy buildings, which we should change cogitation and solve problems from the source.

\section{5 conclusion}

Meteorological architecture makes meteorology the most important part of architectural design and the logical source of architectural space division and architectural form generation. The exploration process of meteorological architecture is a process of thought transition from passive response to active creation in the face of energy crisis. This mode of thinking also provides a lot of inspiration for related fields. Under the background of the new era, when weather has become a new architectural vocabulary to participate in the design of architectural environment, how to establish a stable system model to guide the construction of meteorological buildings is what we need to constantly explore.

\section{Image Source}

Fig.1, Fig.2:http://www.philipperahm.com/data/projects/ convectiveapartments/index-c.html.

Fig.3, Fig.4, Fig.5: https://www.roewu.com/weatherformations

\section{References}

1. Q.Song,L.Yang,B.Yin,HuaZhong Arch, 1003739X04 (2014) -0011-05(2014)

2. L.Li,Time+Arch,1005-684X(2018)03-0036006(2018)

3. X.Lin, Green Building ( China Construction Industry Press, 2011)

4. P.Rahm, Z.Yu, Time+Arch,1005-684X(2015)020032-06 (2015)

5. Y.Qian,J.Zhai,The Architect,107(2019)

6. ROEWU Arch, Weather Formations -An Architectural Meteorology (Garden City Co., Ltd, 2017)

7. Q.Wang,Y.Deng,G.Li,C.Meng,L.Xie,M.Liu,L.Zeng, Chinese Science Bulletin,246(2020) 